\title{
Echinacea verhütet Schnupfen
}

Die Kommission E des deutschen Bundesgesundheitsamtes bescheinigt lediglich dem Presssaft des frischen Krautes vom Purpursonnenhut (Echinacea purpurea) eine Wirksamkeit. Bleibt die Frage, ob Echinacea therapeutisch oder prophylaktisch gegen Erkältungskrankheiten eingesetzt werden sollte.

- In einer randomisierten, placebokontrollierten Doppelblindstudie haben Forscher des "Common Cold Zentrums“ in Cardiff 755 gesunde Versuchspersonen in zwei Gruppen eingeteilt. Die Verumgruppe erhielt vier Monate lang einen standardisierten, alkoholischen Extrakt (2400 mg pro Tag) aus Echinacea purpurea, während die Kontrollgruppe Placebos bekam. Als Studienendpunkte galten diverse Parameter, welche die erlittenen Erkältungen und die Nebenwirkungen quantifizierten. Letztere waren in beiden Gruppen in etwa gleich.

Bezüglich der prophylaktischen Wirksamkeit war Echinacea jedoch dem Placebo signifikant überlegen. In der Placebogruppe wurden insgesamt 850 Erkrankungstage beobachtet, während diese Zahl in der Verumgruppe bei nur 672 lag. Ferner zeigte sich eine Dosis-Wirkungs-Beziehung in dem Sinne, dass Personen, die gute Compliance hatten, auch weniger Erkältungen erlitten. Die Autoren schließen aus diesen Ergebnissen, dass Echinacea bei der Prophylaxe der Erkältung ein positives Nutzen/Risiko-Verhältnis aufweist.

\section{Kommentar}

Das Thema Echinacea wird heiß und kontrovers diskutiert. Eine stattliche Anzahl klinischer Studien steht zu Verfügung, leider sind ihre Resultate jedoch alles andere als einheitlich. Schuld daran ist u. a., dass es eine unüberschaubare Fülle von Präparaten gibt. Nicht nur existieren drei verschiedene Echinaceaspecies, die alle ein differentes Inhaltsprofil haben, es werden zudem verschiedene Planzenteile therapeutisch eingesetzt. Dieses Wirrwarr wird weiter dadurch verkompliziert, dass Echinacea sowohl zur Prophylaxe, wie auch zur Therapie der Erkältung empfohlen wird. Somit ist verständlich, warum die Daten zu diesem Thema unübersichtlich und verwirrend sind. Die vorliegende Studie ist gut gemacht und bestätigt eine ganze Reihe von Voruntersuchungen, die nahelegen, dass Echinacea purpurea wohl die prophylaktisch wirksamste unter den drei Species ist. Die Effektgröße ist nicht gerade umwerfend, aber jeder, der diese Zeilen mit einem Schnupfen liest, wird mir zustimmen: Bei dieser lästigen und schwer zu verhütenden Krankheit ist man selbst mit wenig hoch zufrieden.

\section{- M. Jawad et al.}

Safety and Efficacy Profile of Echinacea purpurea to Prevent Common Cold Episodes: A Randomized, Double-Blind, Placebo-Controlled Trial. Evid. Based. Complement. Alternat. Med. 2012;2012:841315. Epub 2012 Sep 16. 